\title{
The menisci: basic science and advances in treatment
}

\author{
Ehud Rath, John C Richmond
}

\section{Introduction}

Meniscal injury is a major cause of functional impairment of the knee. For many years the meniscus was treated with disrespect as an unnecessary appendage that could be sacrificed with the first hint of malfunction. ${ }^{1}$ In 1948 Fairbank $^{2}$ described radiographic changes following total meniscectomy. As long term results after major meniscectomy were disappointing, a conservative clinical approach to the management of meniscal tears has developed over the past two decades. ${ }^{3-7}$ Meniscal preservation is emphasised, as the menisci play important roles in weight bearing, stabilisation, and energy absorption. In recent decades, a shift towards meniscal preservation has led to the development of new surgical techniques. This article will review the basic science of the semilunar cartilage and current approaches to diagnosis and treatment of meniscal injuries.

\section{Anatomy of the human menisci}

The semilunar cartilage gained its name from the $C$ shape appearance reminiscent of the moon as the month begins. Arising from the tibia, the menisci serve to deepen the surface of the tibial plateau. The menisci are crescents roughly triangular in cross section, covering one half to two thirds of the articular surface of the corresponding tibial plateau. The outer rims of the menisci are convex and attached to the knee joint capsule. The inner edges are concave, thin, and free.

To understand meniscal pathology, it is important to know the basic embryological and vascular features of the meniscus. Both the medial and lateral meniscus assume their characteristic shapes early in prenatal development. ${ }^{8}$ The meniscus is identifiable about seven and half weeks after ovulation. ${ }^{9}$

The blood supply to the menisci originates from the lateral and medial superior and inferior genicular arteries. These vessels reach the periphery of the meniscus through the synovial covering of the anterior and posterior horn attachments. Vessels are present throughout the substance of the fetal menisci. These blood vessels are most prominent in the peripheral one third of the menisci and in the adjacent coronary and capsular ligaments. Beginning at birth, there is a progressive decrease in vascularity proceeding from the inner to the outer regions of the meniscus, although at the age of 10 , vessels can still be identified throughout the inner zones. The adult meniscus is avascular in the inner two thirds. ${ }^{8}{ }^{10}$

The medial meniscus is $\mathrm{C}$ shaped and occupies $50 \%$ of the articular contact area of the medial compartment. Its posterior horn is wider than the anterior horn. The anterior horn is attached firmly to the tibia anterior to the anterior cruciate ligament (ACL). The posterior horn is attached immediately in front of the attachment of the posterior cruciate ligament (PCL). The outer border of the medial meniscus merges with the knee joint capsule. The coronary ligament attaches the meniscus to the upper tibia.

The lateral meniscus is more uniform in width and circular. It covers $70 \%$ of the lateral tibial plateau. The anterior horns of the lateral and medial menisci are attached to each other through the transverse (intermeniscal) ligament. The posterior horn of the lateral meniscus is attached to the PCL and medial femoral condyle through the meniscofemoral ligaments of Wrisberg (the posterior meniscalfemoral ligament) and Humphrey (the anterior meniscal-femoral ligament). It is also attached to the popliteus tendon. ${ }^{11}$ The lateral meniscus is more mobile and is not anchored to the lateral collateral ligament. The anchor of the lateral meniscus to the femur and the popliteal tendon couples its motion with that of the femoral condyle during rotation. It is therefore less likely to be injured than the relatively immobile medial meniscus. In flexion and internal rotation, the popliteal tendon retracts the posterior horn, thus reducing entrapment of the lateral meniscus between the femur and the tibia.

The microanatomy may explain injury patterns. A network of type I collagen fibres arranged in a circumferential direction is the dominant morphological pattern. ${ }^{12}$ Radial oriented fibres may function to restrain motion between the circumferential fibres and resist longitudinal splitting. ${ }^{13}$ Most meniscal tears follow the circumferential pattern.

Magnetic resonance imaging (MRI) studies have shown that the meniscal substance is not always homogeneous. Altered signals do not necessarily represent gross pathology but may be associated with degeneration. ${ }^{13-16}$ This type of abnormal signal was found to be present in $30 \%$ of asymptomatic patients without any history of knee injury. ${ }^{17}$

\section{Lateral meniscal variants}

The lateral meniscus tends to have more developmental variation than the medial meniscus. A discoid shape, once thought to be a result of developmental arrest, was not found by Clark and Ogden $^{8}$ at any stage of development. Kaplan ${ }^{18}$ did not find a discoid meniscus in any of the human fetal knees or other animals that he dissected.

Three types of discoid meniscus have been described: complete, incomplete, and the Wrisberg type..$^{190}$ Both the complete and incomplete types have a firm posterior tibial 
Table 1 Meniscal functions

Tibiofemoral load transmission

Shock absorption

Lubrication

Prevent synovial impingement

Distribute synovial fluid

Contribute to joint stability

Assist in gliding motion

attachment and are considered stable. The Wrisberg variant may have a normal appearance, but it lacks the posterior tibial anchorage. As a result, the meniscofemoral attachment will retract the unrestrained meniscus anteriorly with knee flexion.

The classic symptom of a discoid lateral meniscus is a snapping or popping knee. ${ }^{21}$ The onset is usually in childhood or adolescence and is often atraumatic. The physical findings are generally non-specific. Tenderness is not always localised to the lateral aspect of the knee. A positive McMurray test may suggest meniscal tear.

\section{Meniscal functions and biomechanics}

Long term follow up of meniscectomised knees has shown the important functions of these structures. It has been shown that virtually all menisectomised knees will develop arthritic changes with time. ${ }^{2}{ }^{1322-25}$ The severity of these changes appears to be proportional to the amount of meniscus removed. Laboratory investigations have reinforced these observations..$^{25-27}$ Table 1 summarises some of the important roles of the menisci. Tibiofemoral load transmission, shock absorption, and lubrication are the main functions. ${ }^{28-31}$ The menisci compensate for significant incongruity between the femoral and tibial articulating surfaces. The human menisci transmit $30-55 \%$ of the load in a standing position. ${ }^{28}$ After meniscectomy, tibiofemoral contact area may decrease by $50-70 \%,{ }^{29}{ }^{32}$ leading to a proportional increase in contact stresses. An increase in the trabecular bone density in the proximal tibia has been observed as a result of stress distribution on a smaller area. ${ }^{33}$ These changes will lead eventually to joint degeneration. Menisci contribute significantly to joint lubrication. Some $74 \%$ of the total weight of the meniscus is water. Compression squeezes the liquid out into the joint space, to allow smoother gliding of the joint surfaces. This also helps to distribute synovial fluid throughout the joint and aids the nutrition of the articular cartilage. The menisci contribute to the stability of the knee, largely as secondary soft tissue restraints which prevent anterior tibial displacement. $^{34-36}$

\section{History and clinical presentation of meniscal tear}

A detailed description of the injury and symptoms usually guides the doctor to the diagnosis. Most meniscal tear mechanisms in sport are non-contact in nature and occur while cutting, decelerating, or landing from a jump. With increasing age, because of degeneration of the meniscus, tears often occur with trivial injury. Often the patient is not aware of an injury, only
Clinical tips

- Mechanism of injury-non-contact

- Symptoms and findings are non-specific

- Mechanical symptoms are common

- Assess stability for associated ligament injury

of the symptoms that follow. Mechanical symptoms of popping, catching, locking, or buckling along with joint line pain are suggestive of meniscal tear but are somewhat non-specific. Most meniscal tears lead to a mild synovitis, with swelling for several days after injury, which may be recurrent and activity related.

The patient may recall hearing or feeling a pop at the time of injury. This is highly suggestive of an ACL tear, which is commonly associated with meniscal tearing. Immediate swelling suggests bleeding instead of reactive joint fluid. The patient's lifestyle, activity level, occupation, and previous medical history should be documented, as these factors help to determine treatment after diagnosis is complete. More active patients require more aggressive treatment.

Physical examination of the painful knee starts with inspection of gait pattern. A limp is common and the patient with an acute tear may not be able to bear weight. The presence of asymmetry in thigh muscle mass and effusion are also suggestive. A displaced meniscal tear is often associated with impaired active and passive range of motion, termed "the locked knee". Joint line tenderness is common as well as pain at the terminal degrees of flexion and extension depending on tear location. Special tests to identify meniscal pathology should be used but are not specific. The McMurray test $^{37}$ is performed with the patient supine. With the patient's hip and knee flexed, the examiner alternately internally and externally rotates the foot while palpating the posteromedial or posterolateral joint line respectively. For medial meniscal tearing, the examiner extends the knee with the foot externally rotated. Lateral meniscal tear is assessed by internal foot rotation. The examiner should feel and hear a clunk. A truly positive McMurray test is rare even in proven meniscal tears but has a nearly $100 \%$ specificity. ${ }^{38}$ More commonly the test will elicit pain.

Apley's test is performed with the patient prone. With the knee bent to 90 degrees, the foot is rotated internally and externally, first with distraction and then with compression. A positive test elicits pain with compression. It should be stressed that this test is generally inaccurate and is mentioned to discourage its use. ${ }^{38}$

Several signs are routinely used to assess a patient with suspected meniscal tear. The reliability of the different tests and signs for meniscal lesion has been studied by several authors. ${ }^{39-48}$ Evans et $a l^{40}$ found that a "thud" elicited on the medial joint line was correlated with a medial meniscal tear. A sensitivity of $16 \%$ and specificity of $98 \%$ was reported. This means that only a small percentage of patients 
with meniscal tears present with a positive McMurray test. Evans et al also found that the experience of the examiner had little effect on the accuracy of the test to diagnose medial meniscal tears. Fowler and Lubliner ${ }^{41}$ evaluated the predictive value of common clinical tests for the diagnosis of meniscal tears in 161 patients. Tests used were joint line tenderness, pain on forced flexion, the presence of a positive McMurray test, positive Apley grind, and distraction tests, and the presence of a block to extension. These tests were examined prospectively, and the clinical findings were then compared with arthroscopic findings. The authors have found that no one test was predictive for the diagnosis of a meniscal tear. A combination of several positive tests was highly indicative of a meniscal tear. The stability of the knee should be assessed for concurrent ligamentous injury.

\section{Imaging meniscal pathology}

Plain radiography should be used routinely. The radiographs are inspected for associated skeletal injury, presence of loose bodies, or degenerative changes.

Only two decades ago various arthrographic methods were commonly used for diagnosing both meniscal and cruciate injuries. ${ }^{49} 50$ They have been reported in the radiological literature as an accurate method to demonstrate meniscal lesions. ${ }^{51}$ The method requires sterile preparation and injection of intra-articular contrast medium. After injection, the knee is passively flexed and extended several times with the patient in the supine, sitting, and prone positions. Several radiographic positions are used and the patient is exposed to radiation and risk of infection. Arthrograms are generally reserved for people who are too large for the MRI equipment or for those with claustrophobic symptoms.

MRI is a non-invasive means of obtaining high resolution images of the knee joint without the use of ionising radiation. MRI studies have shown that the meniscal substance is not always homogeneous. Meniscal signals shown by MRI have four grades: 0 , uniformly low signal intensity (normal meniscus); 1, irregular increases in intrameniscal signal; 2 , linear increased signal patterns not extending to the superior or inferior meniscal surface (the clinical importance of this type of change in the meniscal structure, as seen on MRI, is still not well understood); 3, the abnormal signal extends to the articular surface. While grades $0-2$ have no surgical significance, grade 3 is visible by arthroscopy and represents a meniscal tear. ${ }^{52-55}$ One of the reasons for many false positive MRI reports is overinterpretation of grade 2 signals.

Boden et $a l^{17}$ investigated the presence of abnormal MRI findings in 74 asymptomatic patients. The prevalence of MRI findings of a meniscal tear increased from $13 \%$ in patients younger than 45 years of age to $36 \%$ in those older than 45 . Kelly et $a p^{5}$ concluded that false positive results occur more often than false negative results, thus delineating the need to correlate symptoms and clinical and MRI find- ings. Miller ${ }^{43}$ stressed the importance of clinical judgment in the diagnosis of a torn meniscus. In his study, a routine preoperative MRI scan did not improve accuracy over clinical diagnosis alone. Muellner et $a l^{44}$ came to similar conclusions. They evaluated the predictability of clinical examination alone in comparison with MRI in the diagnosis of meniscal tears in competitive athletes. In their study, meniscal lesions were diagnosed on clinical examination and MRI investigation with almost equal accuracy $(89 \%)$. They concluded that the advantages of MRI are the visualisation of the extent of the meniscal tear, and the detection of occult chondral and osseous injuries. Rose and Gold ${ }^{47}$ and Terry $e t a l^{48}$ found equal accuracy for clinical examination and MRI in diagnosing meniscal and ACL tears. They also concluded that MRI, except in certain circumstances, is an expensive and unnecessary diagnostic test in patients with suspected meniscal and ACL pathology. MRI is the modality of choice to evaluate torn discoid lateral meniscus. ${ }^{56}$ Three dimensional axial MR images clearly show intrasubstance and radial tears often associated with discoid meniscus. The diagnostic accuracy with regard to degenerative changes is relatively low, at $67 \% .^{16}$ The cost effectiveness of MRI in the evaluation of meniscal tears must be considered especially when arthroscopic surgery is anticipated.

Meniscal injury in the ACL deficient knee The ACL injured patient is at risk of secondary meniscal tears. Daniel et $a \bar{l}^{7}$ followed 236 patients who had sustained an acute ACL injury. The incidence of late meniscectomy in the ACL injured knee was $20 \%$ over five years of follow up. Only two of 45 patients who had had an early ACL reconstruction required meniscal surgery, an incidence of $4 \%$. They concluded that an ACL reconstruction protects the menisci and is likely to improve the long term function of the joint.

In the ACL deficient knee, the menisci are subjected to anterior-posterior shear forces. Being less mobile, the medial meniscus is more prone to these shear forces and eventual tear. The incidence of meniscal tears at the time of traumatic ACL tear is reported to be from $58 \%$ to $78 \% .^{58}$ The incidence of meniscal tears increases in chronic ACL insufficiency. Meniscal tears in these cases are complex, often have degenerative changes, and are less amenable to suture.$^{59}$ Early ACL reconstruction is therefore strongly recommended for manual labourers or active athletes to preserve the menisci.

\section{Meniscal healing}

The key factor in the process of tissue repair is accessibility of cells and inflammatory mediators to the site of injury. The formation of a clot is an initial phase which provides a scaffold for matrix formation and is a chemotactic stimulus for the cellular elements that are involved in wound healing. ${ }^{60}$ In the adult menisci, only the peripheral $20-30 \%$ is vascular. ${ }^{10}$ This leaves the injured central $70-80 \%$ absent from haematoma and hence with inferior conditions for healing. Webber et al $l^{61}$ showed in tissue culture 


\section{Treatment principles}

- The goal: preserve meniscal tissue

- Non-operative treatment: minor symptoms, less active patients

- Locked knee: urgent treatment

that meniscal cells can proliferate and synthesise an extracellular matrix when exposed to factors that normally present in wound haematoma.

It is important to classify the location of the tear relative to the blood supply of the meniscus. ${ }^{62}$ This allows repair potential of the lesion to be predicted. A red-red tear is located at the meniscal periphery within the vascularised zone or represents capsular detachment. It has the best prognosis for healing, as a functional blood supply persists on the capsular and meniscal sides of the lesion. The red-white tear has no blood supply from the inner surface of the lesion. The remaining peripheral vascularity is usually sufficient for the healing process. A white-white tear is located in the avascular zone and therefore has the least chance to heal.

\section{Treatment options}

On November 1883, Thomas Annandale was the first to suture a medial meniscus. ${ }^{63}$ Half a century later, in 1936, King $^{64}$ showed that degenerative changes will appear in the canine stifle after meniscectomy. He showed that the peripheral meniscal tear has the potential to heal. In the 1950s and 1960s the menisci were discarded as an unnecessary appendage that can be easily removed. ${ }^{1}$ Total meniscectomy was performed for almost any meniscal tear suspected on clinical examination. It took almost a century from Annandale's report until a conservative approach to the management of meniscal tears was applied clinically. In the last two decades, understanding meniscal importance and the development of arthroscopic techniques have improved meniscal preservation. Arthroscopy facilitates identification of meniscal pathology, greatly expands treatment options, and appreciably lowers surgical morbidity.

Surgical treatment is recommended for most meniscal tears except those causing minor symptoms in less active patients. Surgery is urgently indicated for the locked knee. The treatment goal is to preserve as much functional meniscal tissue as possible while addressing the clinical symptoms caused by meniscal tears. A trial of non-operative treatment may be offered in the case of a meniscal tear in a sedentary patient, as symptoms may be minimal or uncommon. Non-operative treatment is usually inadequate for a patient with high physical demands associated with work or sport, as the reduction in activity necessitated by the symptoms is not acceptable.

\section{Current treatment}

Arthroscopy is often necessary to determine the optimal treatment. Partial meniscectomy serves to preserve as much normal meniscus as

\section{Favourable outcome for meniscal} repair

- Time from injury (less than eight weeks)

- Peripheral location of the tear

- Patient age less than 30 years

- Simultaneous ACL reconstruction

possible and is indicated in unstable tears that are not reparable because of location or configuration. The surgeon cautiously removes damaged, pathological, or unstable meniscal tissue, and balances the residual meniscal rim. Stable meniscal tears, especially in the vascular outer area of the meniscus, may be left alone unless no other lesions are present and there is sufficient disability to warrant treatment. ${ }^{65}$

Open, arthroscopically assisted, and totally arthroscopic means have been used for meniscal repair. There are multiple techniques for arthroscopic meniscal repair with suturing, and more recently, the use of bioabsorbable implants. ${ }^{66}$ These implants have significantly reduced the morbidity and complexity of meniscal repair and are leading to an increased number of repairs being performed. While all repaired tears may not heal, lowering the threshold for meniscal repair will considerably reduce the risk of late post-traumatic arthritis.

\section{Long term results and complication of} arthroscopic meniscal repair

The long term success (more than two years follow up) of meniscal repair varies in the literature between 67 and $92 \%,{ }^{7} 67-69$ depending on the type or location of the tear and the means of outcome measurement. Eggli et a $l^{69}$ reported a $27 \%$ overall failure rate after 7.5 years. Most of the failures occurred within the first six months. The factors that were found to influence meniscal healing favourably were time from injury to surgery of less than eight weeks, peripheral location of the tear, patient age less than 30 years, tear length less than 2.5 $\mathrm{cm}$, and tear in the lateral meniscus. In this series, $26 \%$ of the medial meniscal repairs failed, but only $11 \%$ of the lateral meniscal repairs failed. Simultaneous meniscal repair and ACL reconstruction has a higher rate of success, probably because of intra-articular bleeding and fibrin clot formation. DeHaven et $a l^{68}$ showed that the protective effect of the meniscus is preserved after clinically successful repairs in an average 10.9 year follow up study; $85 \%$ of compartments were normal on plane radiographs and only $15 \%$ had grade 1 degenerative changes (sclerosis or mild narrowing measuring 1-2 $\mathrm{mm}$ ).

\section{Meniscal allografts}

Not all meniscal tears are reparable, and resection remains more common than repair. The surgeon needs an alternative for a meniscectomised knee or an unsalvageable meniscus. Meniscal allograft transplantation is a relatively new concept. Dog and sheep models were used in the late 1980s to show the ability of meniscal allograft to heal to a recipient periphery. ${ }^{70}{ }^{71}$ Similar early success has been obtained in 
humans. Meniscal allografts are harvested from young organ donors and are matched by size to the recipient. The most common preservation technique is cryopreservation in glycerol. This allows preservation of donor cell viability. ${ }^{72}$

Currently, the indications for meniscal transplantation are not well defined. Persistent pain in a meniscectomised compartment is the most common indication. Some authors state that the presence of degenerative arthritis is mandatory before considering transplantation. ${ }^{3673}$ Others think that minimal or no arthritic change is ideal. ${ }^{74}$ Considering the fact that meniscal transplantation is relatively new, it is too early to determine its long term impact on the meniscus deficient knee. The reported series are small, and most studied patients have had widely variable associated knee pathologies. In order to determine the effectiveness of this investigational procedure, long term functional and radiographic results are needed. Based on these uncertainties, we have reserved meniscal transplantation in our practice for those patients that have pain after meniscectomy, but little or no degenerative change.

\section{Summary}

Once described as a muscle remnant, and therefore treated with disrespect, the meniscus is now known to be a vital structure within the knee. Minimal partial meniscectomy performed arthroscopically, or meniscal repair when practical, have become the standard treatments. In spite of the advancements in understanding meniscal function and its preservation, much remains to be studied. In the future, availability of better repair techniques and the option of meniscal replacement with allograft or prosthetic menisci are expected to improve outcomes.

1 Larson RL. The knee: the physiological joint [editorial]. $\mathcal{F}$ Bone foint Surg [Am] 1983;65:143-4.

2 Fairbank TJ. Knee joint changes after meniscectomy. 7 Bone foint Surg [Br] 1948;30:664-70.

3 Cameron JC, Saha S. Meniscal allograft transplantation for unicompartmental arthritis of the knee. Clin Orthop 1997 164-71.

4 Fritz JM, Irrgang JJ, Harner CD. Rehabilitation following allograft meniscal transplantation: a review of the literature and case study. F Orthop Sports Phys Ther 1996;24:98-106.

5 Paletta GAJ, Manning T, Snell E, et al. The effect of allograft meniscal replacement on intraarticular contact area and pressures in the human knee. A biomechanical study. $A m \mathcal{F}$ Sports Med 1997;25:692-8.

6 Veltri DM, Warren RF, Wickiewicz TL, et al. Current status of allograft meniscal transplantation. Clin Orthop 1994;4455 .

7 Newman AP, Daniels AU, Burks RT. Principles and decision making in meniscal surgery. Arthroscopy 1993;9: 33-51.

8 Clark CR, Ogden JA. Development of the menisci of the human knee joint. Morphological changes and their potential role in childhood meniscal injury. 7 Bone foint Surg [Am] 1983;65:538-47.

9 Gardner E, O'Rahilly R. The early development of the knee joint in staged human embryos. F Anat 1968;102:289-99.

0 Arnoczky SP, Warren RF. Microvasculature of the human meniscus. Am $\mathcal{F}$ Sports Med 1982;10:90-5.

11 Last RJ. The popliteus muscle and the lateral meniscus. $\mathscr{f}$ Bone foint Surg [Br] 1950;32:93.

12 Bullough P, Goodfellow J. The significance of the fine structure of articular cartilage. F Bone foint Surg $[\mathrm{Br}]$ 1968;50:852

13 Woo SLY, et al. Anatomy, biology and biomechanics of tendon, ligament and meniscus. In: Simon SR, ed. Orthopedic basic science. American Academy of Orthopaedic Surgeons, 1994:45-88.

14 Crues JV, III, Ryu R, Morgan FW. Meniscal pathology. The expanding role of magnetic resonance imaging. Clin Orthop 1990;80-7.

15 Farley TE, Howell SM, Love KF, et al. Meniscal tears: MR and arthrographic findings after arthroscopic repair. Radiology 1991;180:517-22.
16 Raunest J, Oberle K, Loehnert J, et al. The clinical value of manetic resonance imaging in the evaluation of meniscal disorders. F Bone foint Surg [Am] 1991;73:11-16.

17 Boden SD, et al. A prospective and blinded investigation of magnetic resonance imaging of the knee. Abnormal findings in asymptomatic subjects. Clin Orthop 1992;17785.

18 Kaplan EB. Discoid lateral meniscus of the knee joint. Nature, mechanism, and operative treatment. $\mathcal{F}$ Bone foint Surg $[\mathrm{Am}]$ 1957;39:77-87.

19 Jordan MR. Lateral meniscal variants: evaluation and treatment. Fournal of the American Academy of Orthopaedic Surgery 1996; 4:191-200.

20 Watanabe M, Takeda S, Ikeuchi H. Atlas of Arthroscopy. Tokyo: Igaku-Shoin, 1979:75-130.

21 Washington ER, Root L, Liener UC. Discoid lateral meniscus in children. Long term follow-up after excision. F Bone foint Surg [Am] 1995;77:1357-61.

22 Laasonen EM, Wilppula E. Why a meniscectomy fails. Acta Orthop Scand 1976;47:672-5.

23 Noble J, Turner PG. The function, pathology, and surgery of the meniscus. Clin Orthop 1986;62-8.

24 S $\ddagger$ balle K, Hansen AJ. Late results after meniscectomy in children. Injury 1987;18:182-4.

25 Jorgensen U, Sonne-Holm S, Lauridsen F, et al. Long-term follow-up of meniscectomy in athletes a prospective longitudinal study. F Bone foint Surg [Br] 1987;69:80-3.

26 Hede A, Svalastoga E, Reimann I. Articular cartilage changes following meniscal lesions. Repair and meniscectomy studied in the rabbit knee. Acta Orthop Scand 1991;62:319-22.

27 Henning CE, Lynch MA. Current concepts of meniscal function and pathology. Clin Sports Med 1985;4:259-65.

28 Krause WR, Pope MH, Johnson RJ, et al. Mechanical changes in the knee after meniscectomy. F Bone foint Surg [Am] 1976;58:599-604.

29 Kurosawa H, Fukubayashi T, Nakajima H. Load-bearing mode of the knee joint: physical behavior of the knee joint with or without menisci. Clin Orthop 1980;149:28390.

30 Ahmed AM, Burke DL. In-vitro measurement of static pressure distribution in synovial joints. Part I. Tibial surface of the knee. F Biomech Eng 1983;105:216-25

31 Bargar WL, et al. In vivo stability testing of postmeniscectomy knees. Clin Orthop 1980;247-52.

32 Radin EL, deLamotte F, Maquet P. Role of the menisci in the distribution of stress in the knee. Clin Orthop 1984;185: $290-4$.

33 Odgaard A, Pedersen CM, Bentzen SM, et al. Density changes at the proximal tibia after medial meniscectomy. $\mathcal{F}$ Orthop Res 1989;7:744-53.

34 Shoemaker SC, Markolf KL. The role of the meniscus in the anterior-posterior stability of the loaded anterior cruciate deficient knee. Effects of partial versus total exicion. $\mathcal{F}$ Bone foint Surg [Am] 1986;68:71-9.

35 Frank CB, Jackson DW. Current concept review: the science of reconstruction of the anterior cruciate ligament. $\mathcal{F}$ Bone foint Surg [Am] 1997;79:1556-76.

36 Levy IM, Torzilli PA, Warren RF. The effect of medial meniscectomy on anterior-posterior motion of the knee. $\mathcal{F}$ Bone foint Surg [Am] 1982;64:883-8.

37 McMurray TP. The semilunar cartilages. $B r \quad f$ Surg 1942;29:407.

38 Richmond JC. The knee. In: Richmond JC, Shahady EJ, eds. Sports medicine for primary care. Oxford: Blackwell Science, 1996:387-444.

39 Bernstein J, Muellner T. The diagnosis of meniscal tears in athletes. A comparison of clinical and magnetic resonance maging investigations [letter]. Am $\mathcal{F}$ Sports Med 1997;25: $588-9$.

40 Evans PJ, Bell GD, Frank C. Prospective evaluation of the McMurray test. Am f Sports Med 1993;21:604-8.

41 Fowler PJ, Lubliner JA. The predictive value of five clinical signs in the evaluation of meniscal pathology. Arthroscopy 1989;5:184-6.

42 Kim S-J, Min B-H, Han D-Y. Paradoxical phenomena of the McMurray test. An arthroscopic investigation. Am $\mathcal{F}$ Sports Med 1996;24:83-7.

43 Miller GK. A prospective study comparing the accuracy of the clinical diagnosis of meniscus tear with magnetic resonance imaging and its effect on clinical outcome. Arthroscopy 1996;12:406-13.

44 Muellner T, Weinstabl R, Schabus R, et al. The diagnosis of meniscal tears in athletes. A comparison of clinical and magnetic resonance imaging Investigations. Am $\mathcal{7}$ Sports Med 1997;25:7-12.

45 O'Shea KJ, Murphy KP, Heekin RD, et al. The diagnostic accuracy of history, physical examination, and radiographs in the evaluation of traumatic knee disorders. Am F Sports Med 1996;24:164-7

46 Oberlander MA, Shalvoy RM, Hughston JC. The accuracy of the clinical knee examination documented by arthroscopy. A prospective study. Am $\mathcal{F}$ Sports Med 1993;21: $773-8$.

47 Rose NE, Gold SM. A comparison of accuracy between clinical examination and magnetic resonance imaging in the diagnosis of meniscal and anterior cruciate ligament tears. Arthroscopy 1996;12:398-405.

48 Terry GC, Tagert BE, Young MJ. Reliability of the clinical assessment in predicting the cause of internal derangement of the knee. Arthroscopy 1995;11:568-76.

49 Butt WP, McIntyre JL. Double-contrast arthrography of the knee. Radiology 1969;92:487-99. 
50 Pavlov H, Torg JS. Double contrast arthrographic evaluation of the anterior cruciate ligament. Radiology 1978;126: tion of

51 Nicholas JA, Freiberger RH, Killoran PJ. Double contrast arthrography of the knee. Its value in the management of two hundred and twenty-five knee derangements. F Bon foint Surg [Am] 1970;52:203-20.

52 Barronian AD, Zoltan JD, Bucon KA. Magnetic resonance imaging of the knee: correlation with arthroscopy. Arthroscopy $1989 ; 5: 187-91$

53 Crues JV, III, Mink J, Levy TL, et al. Meniscal tears of the knee: accuracy of MR imaging. Radiology 1987;164: 445-8.

54 Kaplan PA, Nelson NL, Garvin KL, et al. MR of the knee: the significance of high signal in the meniscus that does not clearly extend to the surface [see comments]. AfR Am $\mathcal{F}$ Roentgenol 1991;156:333-6.

55 Kelly MA, et al. MR imaging of the knee: clarification of its role. Arthroscopy 1991;7:78-85.

role. Arthroscopy 1991;7:78-85. lateral meniscus. Eur f Radiol 1998;27:153-60.

57 Daniel DM, et al. Fate of the ACL-injured patient. A prospective outcome study. Am $\mathcal{F}$ Sports Med 1994;22:63244.

58 Duncan JB, Hunter R, Purnell M, et al. Meniscal injuries associated with acute anterior cruciate ligament tears in alpine skiers. Am F Sports Med 1995;23:170-2.

59 Keene GCR, Bickerstaff D, Rae PJ, et al. The natural history of meniscal tears in anterior cruciate ligament insufficiency. Am $\mathcal{F}$ Sports Med 1993;21:672-9.

60 Arnoczky SP, Warren RF, Spivak JM. Meniscal repair using an exogenous fibrin clot. An experimental study in dogs. $\mathcal{F}$ Bone foint Surg [Am] 1988;70:1209-17.

61 Webber RJ, Harris MG, Hough AJ, Jr. cell culture of rabbit meniscal fibrochondrocytes: proliferative and synthetic response to growth factors and ascorbate. 7 Orthop Res 1985;3:36-42.
62 De Haven KE, Arnoczky SP. Instructional course lectures. American Academy of Orthopaedic Surgery. Meniscal repair. Part I. Basic science, indication for repair, and open repair. F Bone foint Surg [Am] 1994;76:140-52.

63 Annandale T. An operation for displaced semilunar cartilage. Br F Surg 1885;1:799.

64 King D. The healing of semilunar cartilages. F Bone foint Surg [Am] 1936;18:333-42.

65 Weiss CB, Lundberg M, Hamberg P, et al. Non-operative treatment of meniscal tears. $\mathcal{F}$ Bone foint Surg [Am] $1989 ; 71: 811-22$

66 Jakob RP, Staubli HU, Zuber K, et al. The arthroscopic meniscal repair. Techniques and clinical experience. Am $\mathcal{F}$ Sports Med 1988;16:137-42.

67 Barber FA, Click SD. Meniscus repair rehabilitation with concurrent anterior cruciate reconstruction. Arthroscopy 1997;13:433-7.

68 DeHaven KE, Lohrer WA, Lovelock JE. Long-term results of open meniscal repair. Am f Sports Med 1995;23:524-30.

69 Eggli S, Wegmueller H, Kosina J, et al. Long-term results of arthroscopic meniscal repair. An analysis of isolated tears. Am $\mathcal{F}$ Sports Med 1995;23:715-20.

70 Canham W, Stanish W. A study of the biological behavior of the meniscus as a transplant in the medial compartment of the meniscus as a transplant in the medial compar

71 Milachowski KA, Weismeier K, Wirth CJ. Homologous meniscus transplantation. Experimental and clinical results. Int Orthop 1989;13:1-11.

72 Johnson DL, Bealle D. Meniscal allograft transplantation. Clin Sports Med 1999;18:93-108.

3 Verstraete KL, et al. Current status and imaging of allograft meniscal transplantation. Eur F Radiol 1997;26:16-22.

74 Shelton WR, Treacy SH, Dukes AD, et al. Use of allografts in knee reconstruction. II. Surgical considerations. Fournal of the American Academy of Orthopaedic Surgery 1998;6: $169-75$.

\section{Take home message}

Meniscal tears are common in sports and non-sports related injuries. The long term protective effect of the menisci on the joint surfaces has been well documented. Meniscal preservation should be the goal of treatment. When surgery is indicated, minimally invasive arthroscopic techniques can adequately achieve this goal.

\section{Multiple choice questions}

1 Which of the following accurately illustrates the possibility of meniscal healing?

(a) The medial meniscus covers a wider surface of the tibial plateau

(b) The medial meniscus is attached to the PCL

(c) The lateral meniscus is more mobile

(d) A discoid meniscus is more common on the lateral side

(e) The periphery of the meniscus is vascularised

2 Which of the following is not an important role for the menisci?

(a) Tibiofemoral load transmission

(b) Secondary stabilisers of the knee

(c) Assist patellar tracking

(d) Shock absorption

(e) Distribute joint fluid

3 Which of the following is not a common symptom of meniscal tear?

(a) Anterior knee pain

(b) Catching

(c) Locking

(d) Joint line pain

(e) Recurrent swelling of the joint

4 When compared with a careful history and physical examination of the knee, which of the following statements about MRI is correct?

(a) MRI is much less accurate than clinical evaluation (b) MRI is less accurate than clinical evaluation

(c) MRI has about the same accuracy as clinical evaluation

(d) MRI is more accurate than clinical evaluation

(e) MRI is much more accurate than clinical evaluation

5 In which of the following patients does meniscal repair have the best chance to heal?

(a) 22 year old soccer player with a long standing small tear of the medial meniscus

(b) 17 year old rugby player with an acute combined ACL tear and vascularised lateral meniscal tear

(c) 49 year old carpenter who tore the medial meniscus with a trivial injury one week ago

(d) A torn discoid lateral meniscus in a 15 year old patient with a long history of a "clunk" in the knee

(e) 66 year old woman with atraumatic clicking of recent onset in a knee with osteoarthritis

(Answers p 318.)

\section{Essay questions}

1 Describe the typical clinical presentation of a middle aged worker who tears a meniscus.

2 Why is it important to retain as much healthy meniscus as is possible through early surgery, minimal meniscal resection, and repair if possible? 